Review Article

\title{
ZNF423 and ZNF521: EBF1 Antagonists of Potential Relevance in B-Lymphoid Malignancies
}

\author{
Maria Mesuraca, ${ }^{1}$ Emanuela Chiarella, ${ }^{1}$ Stefania Scicchitano, ${ }^{1}$ Bruna Codispoti, ${ }^{1}$ \\ Marco Giordano, ${ }^{1}$ Giovanna Nappo, ${ }^{1,2}$ Heather M. Bond, ${ }^{1}$ and Giovanni Morrone ${ }^{1}$ \\ ${ }^{1}$ Laboratory of Molecular Haematopoiesis and Stem Cell Biology, Department of Experimental and Clinical Medicine, \\ Magna Groecia University, 88100 Catanzaro, Italy \\ ${ }^{2}$ YCR Cancer Research Unit, Department of Biology, University of York, Heslington, York YO10 5DD, UK
}

Correspondence should be addressed to Heather M. Bond; bond@unicz.it

Received 19 October 2015; Accepted 25 November 2015

Academic Editor: Mariateresa Fulciniti

Copyright (C) 2015 Maria Mesuraca et al. This is an open access article distributed under the Creative Commons Attribution License, which permits unrestricted use, distribution, and reproduction in any medium, provided the original work is properly cited.

\begin{abstract}
The development of the B-lymphoid cell lineage is tightly controlled by the concerted action of a network of transcriptional and epigenetic regulators. EBF1, a central component of this network, is essential for B-lymphoid specification and commitment as well as for the maintenance of the B-cell identity. Genetic alterations causing loss of function of these B-lymphopoiesis regulators have been implicated in the pathogenesis of B-lymphoid malignancies, with particular regard to B-cell acute lymphoblastic leukaemias (B-ALLs), where their presence is frequently detected. The activity of the B-cell regulatory network may also be disrupted by the aberrant expression of inhibitory molecules. In particular, two multi-zinc finger transcription cofactors named ZNF423 and ZNF521 have been characterised as potent inhibitors of EBF1 and are emerging as potentially relevant contributors to the development of B-cell leukaemias. Here we will briefly review the current knowledge of these factors and discuss the importance of their functional cross talk with EBF1 in the development of B-cell malignancies.
\end{abstract}

\section{Introduction}

The specification and development of the diverse blood cell lineages from haematopoietic stem cells have been extensively investigated during the past few decades, leading to substantial advances in our understanding of the regulation of haematopoiesis. In particular, B-lymphopoiesis has been characterised in great detail thanks to the identification of a wealth of molecular and genetic markers that have allowed for the accurate definition of the individual stages of development of the mature B-cell phenotype [1-3]. The B-lymphoid commitment of multipotent haematopoietic progenitors, as well as their progressive lineage restriction, that is, the stepwise acquisition of B-lymphoid features and the parallel loss of alternative developmental potential, is tightly controlled by the concerted action of a complex network of transcriptional and/or epigenetic regulators [2, 4-17]. Among these, early Bcell factor 1 (EBF1) is regarded as a master determinant of the specification, development, and maintenance of the Blymphoid lineage [18].

EBF1 (also termed Olf-1 or COE1, for Collier/Olf-1/EBF1) is the founding member of a family of four DNA-binding proteins implicated in the control of the cell fate choice in multiple tissues [19-24]. In vertebrates, the EBF1 protein is characterised by an $\mathrm{N}$-terminal atypical zinc finger motif that is referred to as "zinc knuckle" [25], responsible for its DNAbinding activity [26] and required for the transcriptional activation of target genes [27], and by an atypical helix-loophelix (HLH) domain, containing duplication of the second helix motif, which mediates dimerisation. Between these domains is an IPT (IG-plexin transcription factor) domain, whose function is uncertain. At the carboxyl-terminal end, $\mathrm{EBF1}$ presents a putative transactivation domain that is largely dispensable for its transcriptional activity [27].

The expression of EBF1 in the haematopoietic system is restricted to the B-lymphoid lineage and is detectable 
from the earliest lymphoid progenitors to mature B-cells and is subjected to complex control. Transcription of the $E B F 1$ gene, controlled by two distinct promoters [28, 29], is initiated in the B-cell biased subset of common lymphoid progenitors by the transcription factors E2A, FOX01, and STAT5 (activated in turn by IL-7R signalling). In later stages of B-cell differentiation, the levels of EBF1 expression are maintained and further enhanced, by a positive feedback loop that involves EBF1 itself and the product of its target gene, PAX5 [29, 30].

The sustained expression of EBF1 is essential in all stages of B-lymphopoiesis [31-33]. Ebf1 gene knockout results in complete lack of B-lymphoid development, accompanied by loss of B-cell-specific gene expression [9]. Conversely, its enforced expression in primitive haematopoietic stem and progenitor cells restricts their differentiation potential to the B-cell lineage [34]. These effects are accomplished both via the transcriptional activation, induced by EBF1 alone or in combination with other factors, of a number of genes crucial for B-cell development (including those encoding EBF1 itself, PAX5, and components of the pre-B-cell receptor such as IGLL1, VPREB, CD79A, and CD79B) and through the repression of genes whose products promote the development of other haematopoietic cell lineages [35]. The latter mechanism is essential not only for lineage restriction, but also for preserving B-lymphoid identity, as indicated by several lines of evidence: conditional knockout of Ebf1 in committed B-cell progenitors results in their conversion to non-B-lineages [33]; haploinsufficiency of Ebf1 alone, or of Ebfl and Runxl, is associated with lineage-promiscuous gene expression in pro- and pre-B-cells [36]; heterozygous deletion of Ebfl and Pax5 induces T-lineage conversion of $\mathrm{CD}_{1}{ }^{+}$pro-B-cells [37]. In immature B-cells, EBF1 strongly inhibits the expression of $B$-limp1, a transcription factor known to repress the Pax5 gene [38]. In addition to its role as a transcriptional activator or repressor, EBF1 possesses properties of an epigenetic regulator and has been shown to initiate chromatin remodelling at the promoter of target genes thereby modulating its accessibility to transcriptional effectors [39-42]. Using a combination of CHIP-seq analyses and of gain- and loss-of-function gene profiling studies, Treiber et al. [11] have shown that EBF1 can induce chromatin remodelling in a set of target loci that poise these genes for expression at later stages of differentiation.

In light of its central role in the network of transcriptional and epigenetic regulators that promote the generation and maintenance of the B-lymphoid phenotype, it is not surprising that perturbations of the expression and/or function of EBF1, especially combined with those of other components of this network, are frequently associated with B-cell malignancies [43-46]. In a murine experimental model, ablation of a single allele of either Ebf1 or Pax5, in combination with a constitutively active version of STAT5b, resulted in the development of B-cell acute lymphoblastic leukaemia (BALL) with complete penetrance [47]. More recently, Ebf1 haploinsufficiency resulting from the insertion of a lentiviral vector in its locus was reported to trigger the occurrence of B-ALL [48]. Ebf1 haploinsufficiency has also been linked to increased susceptibility of pro-B-cells to DNA damage in response to UV light and, though not highly leukaemogenic per se, induced pro-B-ALL development with high frequency when accompanied by Pax5 heterozygosity [49].

The availability of methods that allow genome-wide, high-resolution detection of genetic lesions has led to the discovery of numerous novel genetic alterations that target genes encoding regulators of B-lymphopoiesis in approximately $60 \%$ of B-ALLs [34, 50-55]. Among these, mutations resulting in diminished expression and/or impaired activity of EBF1 are not as common as those affecting PAX5 [51]. Interestingly, however, the frequency of $E B F 1$ deletions was considerably higher in pediatric high-risk B-precursor ALLs [56] and in relapsed ALLs [57], where copy number alterations were detected in $25 \%$ of the cases.

One alternative mechanism through which the activity of EBF1 may be impaired is the inappropriate expression of antagonist factors. Among the known inhibitors of EBF1, two related multi-zinc finger transcription cofactors, zinc finger proteins 423 and 521, have been repeatedly implicated in the development of B-ALL and lymphomas.

\section{Zinc Finger Protein 423}

ZNF423 (also referred to as Olf-1/Ebfl-associated zinc finger protein, OAZ or EBFAZ, and ZFP423 in mouse) is a nuclear protein containing 30 Krüppel-like zinc finger (ZF) motifs, first identified for its ability to bind to OLF-1/EBF1 and to inhibit its transcriptional activation of olfactory-specific genes [58] and to coordinate the expression of immature and mature stage-specific genes in olfactory-receptor neurons where its enforced expression induces maturation arrest [59]. It was determined that the binding between the two factors is mediated by the interaction of the last three zinc fingers of ZNF423/OAZ with the HLH domain of OLF-1/EBF1 [58], and this prevents the generation of transcriptionally active EBF1 homodimers. ZNF423 was also shown to possess direct DNA-binding activity to inverted GCACCCn repeats, mediated by $\mathrm{ZF}$ motifs located in the amino-terminal region of the protein [60]. Subsequent studies showed that, in response to bone morphogenetic protein (BMP) 2, ZNF423 can form complexes with SMAD1 and SMAD4 via its zinc fingers $14-17$ and activate the transcription of BMP target genes [61]. However, the ZNF423-SMAD1/4 complex can also induce transcription of the inhibitory factor, SMAD6, thereby triggering a regulatory loop that limits the intensity and/or duration of BMP signalling [62]. Overexpression of EBF1/OLF-1 was found to modulate the activity of the ZNF423-SMAD1/4 complex, possibly by interfering with its formation through its binding to ZNF423 [61]. Additional relevant interactions of ZFP423 include that with the NOTCH1 intracellular domain, resulting in the selective upregulation of Hes5 expression, which is potentiated by BMPs and antagonised by EBF factors [63]. Binding of ZNF423 with retinoic acid receptors has also been shown to represent an essential molecular partnership [64]. Cho et al. [65] reported the presence of a functional enhancer element containing overlapping EBF1 and ZFP423-binding sites in intron 5 of the $Z f p 423$ gene, whose activity was enhanced by EBF1 but 
strongly suppressed by ZNF423, suggesting the existence of an autoregulatory feedback mechanism.

A wealth of recent experimental evidence has highlighted a central role for ZFP423 in the control of differentiation of adipocyte progenitors [57, 66-68], through the transcriptional activation of PPAR $\gamma$ genes whose products are essential preadipogenic factors. In this process, the activity of ZFP423 is enhanced by BMP4, via SMAD1/4-mediated displacement of WISP2, a WNT-induced adipokine that sequesters ZFP423 in the cytoplasm [69]. The proadipogenic effect of EBF1 has been in part ascribed to the stimulation of $Z f p 423$ expression in mesenchymal progenitors [70].

Finally, ZNF423 has been implicated in CNS midline patterning, vermis formation, and cerebellar development [59, $71,72]$, in DNA damage response and ciliogenesis (through its interactions with the poly-ADP ribosyl polymerase $1[62,73]$ and the centrosomal/cilia protein CEP290 [73]), and in the transcriptional regulation of BRCA1 [74].

\section{Zinc Finger Protein 521}

ZNF521/ZFP521 is the paralogue of ZNF423/ZFP423, and like ZNF423, it contains 30 Krüppel-like zinc fingers, and at the $\mathrm{N}$-terminal end it harbours a 12 -amino acid motif (NBD). This motif is shared with a number of transcriptional corepressors and recruits the nucleosome remodelling and histone deacetylase (NuRD) complex [75-77]. In ZNF521, the NBD is encoded by a short exon, raising the possibility that alternative splicing may generate a variant protein unable to bind the NuRD, whereas the NBD-containing isoform of ZNF423 is generated by the activation of an alternative upstream promoter [78].

Zfp521 was originally identified as a common target gene for retroviral integration associated with the occurrence of Bcell lymphomas in AKXD mice and hence termed ecotropic viral integration site 3 (Evi3) [71]. The cDNA encoding human ZNF521 (initially designated early hematopoietic zinc finger protein, EHZF) was cloned for its abundant and selective expression in primitive haematopoietic progenitors [75]. Within the haematopoietic system, ZNF521 expression is almost completely restricted to stem and early progenitor cells [75, 76, 78-81]. Like ZNF423, this factor has been shown to cooperate with SMAD1/4 in the transcriptional activation of BMP target genes [75] and to strongly inhibit the expression of B-cell-specific EBF1 target genes with a mechanism that is largely independent of the NuRD complex recruitment [75, 82]. Silencing of ZNF521 in human and murine haematopoietic progenitors considerably enhances the production of B-cells in vitro [82]. This suggests that ZNF521 counteracts the activity of EBF1 and other transcription factors that promote differentiation of haematopoietic progenitors such as GATA1 [83] and may contribute to the homeostasis of the immature haematopoietic cell compartment. Recently, using a mathematical model based on relevant literature to define key molecular interactions in the transcriptional network that governs B-lymphopoiesis, Salerno et al. [84] have identified the balance between EBF1 and ZNF521 as one major factor in B-lymphoid specification. According to this model, a shift of this balance toward
ZNF521 is predicted to result in dedifferentiation of B-cell progenitors.

In addition to the haematopoietic system, the interplay between ZNF521 and EBF1 appears to be relevant in the determination of cell fate in other systems, including the developing striatum [85] and mesenchymal progenitors. In the latter, ZFP521 inhibits the proadipogenic activity of EBF1 and represses the EBF1-induced expression of $Z f p 423$, acting both on the intronic enhancer and at the level of the $Z f p 423$ promoter, thereby favouring osteoblastic commitment at the expense of adipogenesis [70, 86]. Zfp521 is in turn repressed by EBF1 [70]. In osteoblasts, ZFP521 stimulates bone formation by antagonising both RUNX2 $[87,88]$ and EBF1 [89]; in addition, ZFP521-mediated inhibition of EBF1 was reported to modulate both the intrinsic and osteoblastdependent osteoclastogenesis [89]. Human articular chondrocytes appear to require ZNF521 for the maintenance of their identity, and ZNF521 silencing results in a markedly dedifferentiated phenotype when these cells are cultured in alginate beads [90]. Whether EBF1 contributes to this phenomenon remains yet to be determined.

A property of ZNF521 potentially relevant to cancer was discovered by La Rocca et al. [91] who showed that enforced expression of ZNF521 enhances HLA Class I expression on the tumour cell surface, with particular regard to multiple myeloma cells, thereby preventing their recognition by natural killer cells.

A growing body of evidence has also delineated a prominent role for ZNF521/ZFP521 as a regulator of neurogenesis. Kamiya et al. [92] showed that ZFP521 promotes the spontaneous transition of epiblasts to neuroectodermal progenitors, through the activation of early neural genes in a process that requires the interaction of ZFP521 with the coactivator P300. ZNF521 transcript is abundant in the brain [75], particularly in neural stem cells and cerebellar granule neuron precursors [76], which are considered the cells of origin of a substantial fraction of medulloblastomas, the most common malignant brain tumours in children. Consistently, ZNF521 has been shown to stimulate the growth, clonogenicity, and tumorigenicity of human and murine medulloblastoma stem-like cells [93]. Unlike Zfp423, Zfp521 knockout does not appear to dramatically disrupt cerebellar development but results in behavioural abnormalities and in the reduction in the number of neuronal progenitors in the dentate gyrus and in cerebellum [94]. Finally, a recent report has documented the existence of an incoherent feed-forward loop in which the RUNX1-induced expression of $Z f p 521$ in a subset of RUNX1dependent sensory neurons activates gene expression programmes that lead to the development of $\mathrm{VGLUT3}^{+}$lowthreshold c-mechanoreceptors while repressing genes driving the choice of alternative cell fates [95].

\section{ZNF423 and ZNF521 in B-Lymphoid Malignancies}

As highlighted in the previous section, Zfp521/Evi3 was initially discovered because its dysregulated expression, induced by retroviral insertion, was associated with the development 
of pre-B- or B-cell lymphomas in AKXD mice [71, 96]. A subsequent study [97] detected constitutive expression of Zfp423/Ebfaz (normally not expressed in haematopoietic cells) as a consequence of another frequent viral integration in AKXD-27 B-cell lymphomas. The integration in Ebfaz and in Evi3 was mutually exclusive, suggesting functional redundancy of these two candidate oncogenes. In light of the shared EBF1-inhibitory activity of ZFP423 and ZFP521, it is conceivable that dysregulated expression of these factors might contribute to the development of B-cell malignancies. More recently, Hiratsuka et al. [98] reported that overexpression of $Z f p 521$ in SL/Kh mice, due to retroviral insertion in its locus, caused the upregulation of pre-BCR-associated signalling molecules, including BANK1, BLNK, and BTK. In the presence of concomitant viral integration targeting other regulatory genes such as $c-M y c, Z f p 521$ overexpression may eventually give rise to pre-B-cell lymphomas in these mice. It must be taken into account that the genetic background of AKXD-27 and SL/Kh mice, both prone to lymphoma development, may be relevant in determining the phenotypes observed in these studies.

Hiratsuka et al. [98] also detected expression of ZNF521 protein in human B-cell lymphoblastic lymphomas. It is puzzling, however, that the localisation of ZNF521 in these cells appeared to be predominantly cytoplasmic, raising the issue of potential staining artifacts. It will be interesting, in future studies, to assess whether aberrant expression of ZNF521 in human lymphoma cells can be confirmed by gene profiling, in situ hybridisation, or mass-spectrometry-based proteomic analyses.

Hentges et al. [99] observed that upregulated expression of Evi3 in aged female AKXD-27 mice was associated with the occurrence of B-lymphoid neoplasias resembling pro-Bcell leukaemias. In addition to overexpressing Zfp521/Evi3, the malignant cells displayed marked upregulation of Ebfl and of its target genes. Based on these data, it was postulated that ZFP521 may antagonise, or synergise with, EBF1 in a cell-type-specific manner [99]. This hypothesis was not confirmed by our subsequent investigation conducted in Bcells, where ZNF521 effectively repressed the expression of EBF1 target genes [82], and remains to be validated. However, a link between aberrant expression of Zfp521 or of $Z f p 423$ and development of B-cell precursor leukaemias is supported by diverse experimental in vivo models of leukaemogenesis based on mice engineered to generate mutation backgrounds that mimic those associated with B-ALLs (reviewed in [100]). In an attempt to identify factors that cooperated with BCR$\mathrm{ABL}$ to induce the progression of chronic myeloid leukaemia, Miyazaki et al. [101] used transgenic BCR-ABL P210 mice crossed with BXH2 mice, which transmit a replicationcompetent retrovirus. They found that constitutive expression of $Z f p 423$, resulting from viral integration in its $5^{\prime}$ noncoding region, led to the development of a B-lineage blast crisis with early onset. This was further supported by the detection of high expression of ZNF423 in cells from CML patients with B-lymphoid blast crisis, but not those in chronic phase [101]. van der Weyden et al. [102] generated a B-ALL mouse model in which the expression of the ETV6-RUNX1 fusion gene (derived from the $\mathrm{t}(12 ; 21)(\mathrm{p} 13 ; \mathrm{q} 22)$ translocation, the most common chromosomal rearrangement in B-ALLs) was combined to Pax5 haploinsufficiency. Transposon-mediated insertional mutagenesis was then performed to identify cooperating B-ALL driver genes and led to the identification of five transposon common insertion sites, including one in the Zfp423 gene, which was associated with a significant increase in the occurrence of B-cell precursor ALLs in these mice [102]. In a similar approach, Yamasaki et al. [103] sought to identify cooperating drivers for the E2A$H L F$ fusion gene generated by the $t(7 ; 19)$ translocation, whose rare occurrence characterises ALLs with extremely poor prognosis, by retroviral-mediated insertional mutagenesis in an E2A-HLF knock-in mouse. One of the three common integration sites identified in this study and associated with B-ALL development lay in the $Z f p 521$ locus. To confirm these findings, the authors generated transgenic mice with enforced expression of $Z f p 521$ in lymphoid cells, crossed them with $E 2 A-H L F$ knock-in animals, and detected B-ALLs in $50 \%$ of the offspring but not in the parental mice [103].

Thus, several lines of experimental evidence suggest that ZFP423 and ZFP521 may cooperate with oncogenic lesions and contribute to B-ALL development, presumably through the inhibition of EBF1 and the consequent disruption of the functional network that governs normal B-cell differentiation. This notion is also supported by the results of some studies of human B-ALLs. As mentioned above, Miyazaki et al. [101] detected abundant levels of ZNF423 transcript in patients with CML blast crisis, but not in those in chronic phase; more recently, a gene profiling analysis of human BALLs detected aberrant expression of ZNF423 in most of the cases studied and established a significant correlation between high expression levels and adverse outcome in ETV6-RUNX1-negative B-ALLs [78]. The analysis of publicly available datasets, conducted and visualised using Oncomine (Compendia Bioscience, Ann Arbor, MI), confirmed that abundant ZNF423 expression is typically found in B- and, to a lesser extent, in T-ALLs. However, this does not appear to be the case for ZNF521, whose expression is relatively high in a significant fraction of AMLs and T-ALLs, but (apart from rare instances, such as $\operatorname{dic}(9 ; 18)(\mathrm{p} 13 ; \mathrm{q} 11)$ translocation in which its gene is fused with that encoding PAX5 and the expression of the resulting chimeric gene is driven by the $\mathrm{B}$-lymphoid PAX5 promoter [51]) is distinctly low or undetectable in virtually all B-ALLs (Figure 1; [76, 78] Mesuraca, in preparation).

This is consistent with the data of Aibar et al. [105], who designed and used an R package named geNetClassifier to discover subsets of genes that unequivocally differentiate and classify different leukaemia subtypes (cALL/pre-B-ALL, AML, CLL, and CML). In this study, ZNF423 was second top ranking in a cohort of 799 genes whose expression is characterised as ALL specific, whereas ZNF521 ranked sixth among 213 AML-specific genes.

How can the apparent lack of ZNF521 expression in ALLs be reconciled with its proposed role as a driver in these leukaemias? One possible clue is offered by a recent report by Aoki et al. [106]. These authors investigated the leukaemiainitiating cells (LICs), a rare subpopulation of leukaemic cells endowed with stem-like features, capable of initiating leukaemia if transplanted into immunocompromised 
ZNF423 expression in Haferlach leukaemia Acute lymphoblastic leukaemia type: B-cell acute lymphoblastic leukaemia Haferlach leukaemia statistics Overexpression gene rank: 1925 (in top 10\%) Reporter: 214761_at

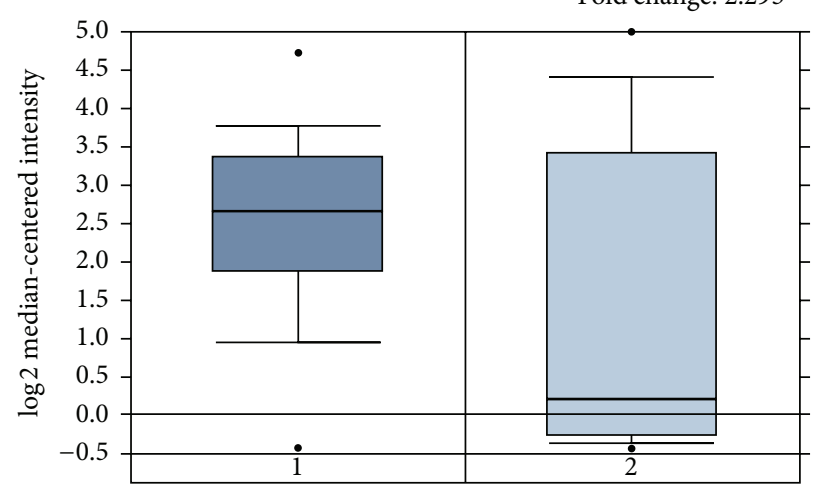

(1) B-cell acute lymphoblastic leukaemia (576)

(2) T-cell acute lymphoblastic leukaemia (174)
ZNF521 expression in Haferlach leukaemia Acute lymphoblastic leukaemia type: B-cell acute lymphoblastic leukaemia Haferlach leukaemia statistics $\begin{array}{ll}\text { Underexpression gene rank: } 984 \text { (in top 6\%) } & P \text { value: } 5.36 E-20 \\ \text { Reporter: 226676_at } & t \text {-test: }-10.098 \\ & \text { Fold change: }-2.105\end{array}$

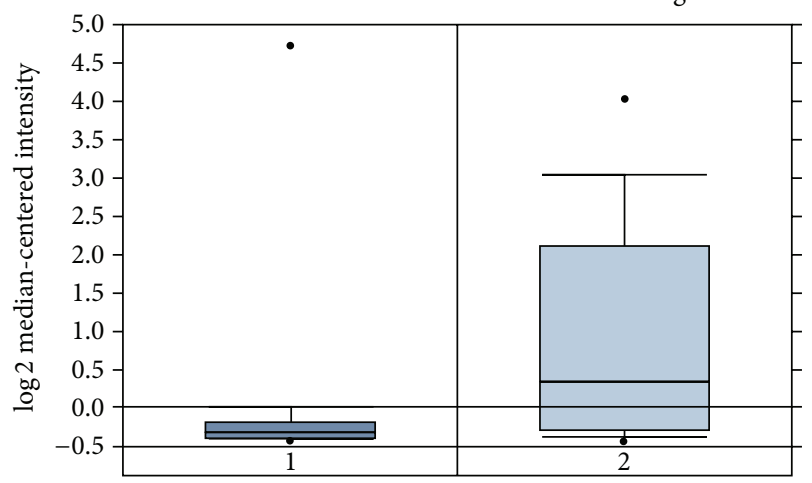

(1) B-cell acute lymphoblastic leukaemia (576)

(2) T-cell acute lymphoblastic leukaemia (174)

(b)

Figure 1: Expression of ZNF423 and ZNF521 in B- and T-ALLs. The Oncomine database was queried for the expression of ZNF423 and ZNF521 in DNA microarray studies of acute lymphoblastic leukaemias. The data shown are from [104] and document the overexpression of ZNF423 and the underexpression of ZNF521 in B-ALLs, whereas both genes display detectable expression in the T-ALLs studied.

animals, in B-ALLs bearing different rearrangements of the $M L L$ gene. In particular, they determined that the LIC fraction of ALLs carrying the $\mathrm{t}(9 ; 11)$ translocation, which generates the $M L L-A F 9$ fusion oncogene, was contained in the $\mathrm{CD} 34^{-} / \mathrm{CD} 19^{+}$cell subset. A gene profiling analysis revealed that ZNF521 was one of the genes whose expression was selectively enriched in these cells. Thus, aberrant expression of ZNF521, occurring in the LIC subset but not necessarily present in the bulk of leukaemic cells, may contribute to the development of some B-ALLs while remaining undetectable when the transcriptome of the whole leukaemic cell population is analysed.

ZNF521 is among the top 25 genes overexpressed in AMLs with $M L L$ fusion genes [107], in particular those expressing MLL-AF9 ([76]; Mesuraca in preparation), and is recognised as one of the prominent downstream targets of MLL-AF9 in AML cells [108]. Its expression may be activated by the AF9 moiety of the fusion protein via an epigenetic mechanism that involves the recruitment of 5-methylcytosine dioxygenase TET2, as it has been observed during the induction of neural differentiation of human ES cells [109]. MLL-AF9-transformed haematopoietic stem cells can give rise to myeloid or lymphoid leukaemias based on their intrinsic developmental potential and on signals provided by the microenvironment [110-112]. Intriguingly, overexpression of ZNF521 was detected in CD34 $4^{+}$cells transformed in vitro by MLL-AF9 and cultured in both myeloid and lymphoid conditions [112]. It could thus be hypothesized that if the MLL-AF9 ${ }^{+}$LICs follow the B-ALL pathway, the expression of ZNF521 is progressively attenuated by B-lymphoid regulatory factors that are known to repress its transcription including IKAROS [113], EBF1 [70], and possibly PAX5 [84]. Conversely, in MLL-AF9 ${ }^{+}$AMLs, the sustained expression of ZNF521 is ensured by the fusion oncoprotein in the presence of a permissive molecular context. Whether the presence of ZNF521 in ALL leukaemia-initiating cells is limited to those expressing MLL-AF9 or is a more general feature remains to be established.

A different scenario applies to ZNF423, whose expression is normally absent in the haematopoietic system. In their study, Harder et al. [78] determined that inappropriate expression of ZNF423 was driven by the removal of epigenetic barriers, namely, demethylation of regulatory elements that normally prevent its expression in the haematopoietic system, combined with the transcriptional induction mediated by BMP2 whose expression is also upregulated in B-ALLs. Alternatively, aberrant expression of ZNF423 in LICs may result from copy number gain, secondary to genomic instability caused by ROS-induced oxidative DNA damage, as observed by Bolton-Gillespie et al. [114] in a murine model of imatinib-refractory CML. However it is initiated, the sustained expression of ZNF423 may then be maintained also by the positive transcriptional effect of $\operatorname{EBF} 1[65,70]$.

\section{Conclusions and Perspectives}

Taken together, the evidence reviewed above indicates that aberrant expression of ZNF423 and ZNF521, triggered by diverse mechanisms, may contribute to the pathogenesis of B-lymphoid malignancies by perturbing the activity of EBF1, a central component of the regulatory network that governs normal B-lymphopoiesis. Our knowledge of the biological 
properties of these two factors is still incomplete and several questions remain, such as whether the repression of EBF1 target genes is the only mechanism responsible for their proleukaemogenic effect, the extent to which their expression contributes to the transformation of B-cell progenitors, and the role of epigenetic modifiers (e.g., the NuRD complex) that both proteins are able to recruit through their N-terminal domain, as well as other molecular partners of ZNF423 and ZNF521. Future studies addressing these issues will further our understanding of the biological and clinical relevance of ZNF423 and ZNF521 in the pathogenesis of B-ALLs and of their potential value as candidate molecular targets for therapeutic intervention.

\section{Conflict of Interests}

The authors declare that there is no conflict of interests regarding the publication of this paper.

\section{Acknowledgments}

Experimental work carried out in the authors' laboratory and described in this paper was supported by funds from the Italian Association for Cancer Research (AIRC) and by the PON01_2834 Prometeo project. Stefania Scicchitano, Bruna Codispoti, and Giovanna Nappo were supported by fellowships of the Ph.D. Programme in Molecular Oncology; Emanuela Chiarella was supported by a PON03PE_00009_2 "ICARE" postdoctoral fellowship; Marco Giordano was supported by a POR Calabria FSE 1007-2013 “HEMMAS” postdoctoral fellowship. The authors are most grateful to Dr. Luca Salerno for the invaluable help with graphics.

\section{References}

[1] R. R. Hardy and K. Hayakawa, "B cell development pathways," Annual Review of Immunology, vol. 19, pp. 595-621, 2001.

[2] R. R. Hardy, P. W. Kincade, and K. Dorshkind, "The protean nature of cells in the B lymphocyte lineage," Immunity, vol. 26, no. 6, pp. 703-714, 2007.

[3] R. S. Welner, R. Pelayo, and P. W. Kincade, "Evolving views on the genealogy of B cells," Nature Reviews Immunology, vol. 8, no. 2, pp. 95-106, 2008.

[4] E. V. Rothenberg, "Stepwise specification of lymphocyte developmental lineages," Current Opinion in Genetics and Development, vol. 10, no. 4, pp. 370-379, 2000.

[5] J. Hagman and K. Lukin, "Transcription factors drive B cell development," Current Opinion in Immunology, vol. 18, no. 2, pp. 127-134, 2006.

[6] C. V. Laiosa, M. Stadtfeld, and T. Graf, "Determinants of lymphoid-myeloid lineage diversification," Annual Review of Immunology, vol. 24, pp. 705-738, 2006.

[7] A. Schebesta, S. McManus, G. Salvagiotto, A. Delogu, G. A. Busslinger, and M. Busslinger, "Transcription factor Pax5 activates the chromatin of key genes involved in B cell signaling, adhesion, migration, and immune function," Immunity, vol. 27, no. 1, pp. 49-63, 2007.

[8] S. L. Nutt and B. L. Kee, "The transcriptional regulation of B cell lineage commitment," Immunity, vol. 26, no. 6, pp. 715-725, 2007.
[9] H. Lin and R. Grosschedl, "Failure of B-cell differentiation in mice lacking the transcription factor EBF," Nature, vol. 376, no. 6537, pp. 263-267, 1995.

[10] E. M. Mandel and R. Grosschedl, "Transcription control of early B cell differentiation," Current Opinion in Immunology, vol. 22, no. 2, pp. 161-167, 2010.

[11] T. Treiber, E. M. Mandel, S. Pott et al., "Early B cell factor 1 regulates $\mathrm{B}$ cell gene networks by activation, repression, and transcription- independent poising of chromatin," Immunity, vol. 32, no. 5, pp. 714-725, 2010.

[12] T. Yoshida, S. Y.-M. Ng, and K. Georgopoulos, "Awakening lineage potential by Ikaros-mediated transcriptional priming," Current Opinion in Immunology, vol. 22, no. 2, pp. 154-160, 2010.

[13] T. Yokota, T. Sudo, T. Ishibashi et al., "Complementary regulation of early B-lymphoid differentiation by genetic and epigenetic mechanisms," International Journal of Hematology, vol. 98, no. 4, pp. 382-389, 2013.

[14] M. A. Choukrallah and P. Matthias, “The interplay between chromatin and transcription factor networks during B cell development: who pulls the trigger first?" Frontiers in Immunology, vol. 5, article 156, 2014.

[15] C. Dege and J. Hagman, "Mi-2/NuRD chromatin remodeling complexes regulate B and T-lymphocyte development and function," Immunological Reviews, vol. 261, no. 1, pp. 126-140, 2014.

[16] R. Grosschedl, "Establishment and maintenance of B cell identity," Cold Spring Harbor Symposia on Quantitative Biology, vol. 78, no. 1, pp. 23-30, 2013.

[17] E. V. Rothenberg, "Transcriptional control of early T and B cell developmental choices," Annual Review of Immunology, vol. 32, pp. 283-321, 2014.

[18] S. Boller and R. Grosschedl, "The regulatory network of B-cell differentiation: a focused view of early B-cell factor 1 function," Immunological Reviews, vol. 261, no. 1, pp. 102-115, 2014.

[19] S. Garel, F. Marín, R. Grosschedl, and P. Charnay, "Ebf1 controls early cell differentiation in the embryonic striatum," Development, vol. 126, no. 23, pp. 5285-5294, 1999.

[20] L. Dubois and A. Vincent, "The COE-Collier/Olf1/EBFtranscription factors: structural conservation and diversity of developmental functions," Mechanisms of Development, vol. 108, no. 1-2, pp. 3-12, 2001.

[21] P. Åkerblad, U. Lind, D. Liberg, K. Bamberg, and M. Sigvardsson, "Early B-cell factor (O/E-1) is a promoter of adipogenesis and involved in control of genes important for terminal adipocyte differentiation," Molecular and Cellular Biology, vol. 22, no. 22, pp. 8015-8025, 2002.

[22] D. Liberg, M. Sigvardsson, and P. Åkerblad, "The EBF/Olf/Collier family of transcription factors: regulators of differentiation in cells originating from all three embryonal germ layers," Molecular and Cellular Biology, vol. 22, no. 24, pp. 8389-8397, 2002.

[23] M. Garcia-Dominguez, C. Poquet, S. Garel, and P. Charnay, "Ebf gene function is required for coupling neuronal differentiation and cell cycle exit," Development, vol. 130, no. 24, pp. 6013-6025, 2003.

[24] D. G. T. Hesslein, J. A. Fretz, Y. Xi et al., "Ebf1-dependent control of the osteoblast and adipocyte lineages," Bone, vol. 44, no. 4, pp. 537-546, 2009.

[25] J. Hagman, M. J. Gutch, H. Lin, and R. Grosschedl, "EBF contains a novel zinc coordination motif and multiple dimerization and transcriptional activation domains," The EMBO Journal, vol. 14, no. 12, pp. 2907-2916, 1995. 
[26] A. Travis, J. Hagman, L. Hwang, and R. Grosschedl, "Purification of early-B-cell factor and characterization of its DNAbinding specificity," Molecular and Cellular Biology, vol. 13, no. 6, pp. 3392-3400, 1993.

[27] S. Fields, K. Ternyak, H. Gao, R. Ostraat, J. Akerlund, and J. Hagman, "The 'zinc knuckle' motif of early B cell factor is required for transcriptional activation of B cell-specific genes," Molecular Immunology, vol. 45, no. 14, pp. 3786-3796, 2008.

[28] E. M. K. Smith, R. Gisler, and M. Sigvardsson, "Cloning and characterization of a promoter flanking the early B cell factor $(\mathrm{EBF})$ gene indicates roles for E-proteins and autoregulation in the control of EBF expression," Journal of Immunology, vol. 169, no. 1, pp. 261-270, 2002.

[29] S. Roessler, I. Györy, S. Imhof et al., "Distinct promoters mediate the regulation of Ebfl gene expression by interleukin-7 and Pax5," Molecular and Cellular Biology, vol. 27, no. 2, pp. 579-594, 2007.

[30] M. Fuxa, J. Skok, A. Souabni, G. Salvagiotto, E. Roldan, and M. Busslinger, "Pax5 induces V-to-DJ rearrangements and locus contraction of the immunoglobulin heavy-chain gene," Genes and Development, vol. 18, no. 4, pp. 411-422, 2004.

[31] S. Zandi, R. Mansson, P. Tsapogas, J. Zetterblad, D. Bryder, and M. Sigvardsson, "EBF1 is essential for B-lineage priming and establishment of a transcription factor network in common lymphoid progenitors," Journal of Immunology, vol. 181, no. 5, pp. 3364-3372, 2008.

[32] B. Vilagos, M. Hoffmann, A. Souabni et al., "Essential role of EBF1 in the generation and function of distinct mature B cell types," Journal of Experimental Medicine, vol. 209, no. 4, pp. 775-792, 2012.

[33] R. Nechanitzky, D. Akbas, S. Scherer et al., "Transcription factor EBF1 is essential for the maintenance of B cell identity and prevention of alternative fates in committed cells," Nature Immunology, vol. 14, no. 8, pp. 867-875, 2013.

[34] Z. Zhang, C. V. Cotta, R. P. Stephan, C. G. de Guzman, and C. A. Klug, "Enforced expression of EBF in hematopoietic stem cells restricts lymphopoiesis to the B cell lineage," The EMBO Journal, vol. 22, no. 18, pp. 4759-4769, 2003.

[35] J. M. R. Pongubala, D. L. Northrup, D. W. Lancki et al., "Transcription factor EBF restricts alternative lineage options and promotes B cell fate commitment independently of Pax5," Nature Immunology, vol. 9, no. 2, pp. 203-215, 2008.

[36] K. Lukin, S. Fields, L. Guerrettaz et al., "A dose-dependent role for EBF1 in repressing non-B-cell-specific genes," European Journal of Immunology, vol. 41, no. 6, pp. 1787-1793, 2011.

[37] J. Ungerback, J. Ahsberg, T. Strid, R. Somasundaram, and M. Sigvardsson, "Combined heterozygous loss of Ebf1 and Pax5 allows for T-lineage conversion of B cell progenitors," Journal of Experimental Medicine, vol. 212, no. 7, pp. 1109-1123, 2015.

[38] H. Kikuchi, M. Nakayama, Y. Takami, F. Kuribayashi, and T. Nakayama, "EBF1 acts as a powerful repressor of Blimp-1 gene expression in immature B cells," Biochemical and Biophysical Research Communications, vol. 422, no. 4, pp. 780-785, 2012.

[39] H. Maier, R. Ostraat, H. Gao et al., "Early B cell factor cooperates with Runxl and mediates epigenetic changes associated with mb-1 transcription," Nature Immunology, vol. 5, no. 10, pp. 10691077, 2004.

[40] J. Hagman and K. Lukin, "Early B-cell factor 'pioneers' the way for B-cell development," Trends in Immunology, vol. 26, no. 9, pp. 455-461, 2005.

[41] H. Gao, K. Lukin, J. Ramírez, S. Fields, D. Lopez, and J. Hagman, "Opposing effects of SWI/SNF and Mi-2/NuRD chromatin remodeling complexes on epigenetic reprogramming by EBF and Pax5," Proceedings of the National Academy of Sciences of the United States of America, vol. 106, no. 27, pp. 11258-11263, 2009.

[42] J. Hagman, J. Ramírez, and K. Lukin, "B lymphocyte lineage specification, commitment and epigenetic control of transcription by early B cell factor 1," Current Topics in Microbiology and Immunology, vol. 356, pp. 17-38, 2012.

[43] E. Campos-Sanchez, A. Toboso-Navasa, I. Romero-Camarero, M. Barajas-Diego, I. Sanchez-Garcia, and C. Cobaleda, "Acute lymphoblastic leukemia and developmental biology: a crucial interrelationship," Cell Cycle, vol. 10, no. 20, pp. 3473-3486, 2011.

[44] S. H. M. Pang, S. Carotta, and S. L. Nutt, "Transcriptional control of pre-B cell development and leukemia prevention," Current Topics in Microbiology and Immunology, vol. 381, pp. 189-213, 2014.

[45] P. Pérez-Vera, A. Reyes-León, and E. M. Fuentes-Pananá, "Signaling proteins and transcription factors in normal and malignant early B cell development," Bone Marrow Research, vol. 2011, Article ID 502751, 10 pages, 2011.

[46] R. Somasundaram, M. A. Prasad, J. Ungerback, and M. Sigvardsson, "Transcription factor networks in B-cell differentiation link development to acute lymphoid leukemia," Blood, vol. 126, no. 2, pp. 144-152, 2015.

[47] L. M. Heltemes-Harris, M. J. L. Willette, L. B. Ramsey et al., "Ebf1 or Pax5 haploinsufficiency synergizes with STAT5 activation to initiate acute lymphoblastic leukemia," Journal of Experimental Medicine, vol. 208, no. 6, pp. 1135-1149, 2011.

[48] D. Heckl, A. Schwarzer, R. Haemmerle et al., "Lentiviral vector induced insertional haploinsufficiency of EBF1 causes murine leukemia," Molecular Therapy, vol. 20, no. 6, pp. 1187-1195, 2012.

[49] M. A. Prasad, J. Ungerback, J. Ahsberg et al., "Ebf1 heterozygosity results in increased DNA damage in pro-B cells and their synergistic transformation by Pax5 haploinsufficiency," Blood, vol. 125, no. 26, pp. 4052-4059, 2015.

[50] R. P. Kuiper, E. F. P. M. Schoenmakers, S. V. van Reijmersdal et al., "High-resolution genomic profiling of childhood ALL reveals novel recurrent genetic lesions affecting pathways involved in lymphocyte differentiation and cell cycle progression," Leukemia, vol. 21, no. 6, pp. 1258-1266, 2007.

[51] C. G. Mullighan, S. Goorha, I. Radtke et al., "Genomewide analysis of genetic alterations in acute lymphoblastic leukaemia," Nature, vol. 446, no. 7137, pp. 758-764, 2007.

[52] C. G. Mullighan, C. B. Miller, I. Radtke et al., "BCR-ABL1 lymphoblastic leukaemia is characterized by the deletion of Ikaros," Nature, vol. 453, no. 7191, pp. 110-114, 2008.

[53] S. Shah, K. A. Schrader, E. Waanders et al., "A recurrent germline PAX5 mutation confers susceptibility to pre-B cell acute lymphoblastic leukemia," Nature Genetics, vol. 45, no. 10, pp. 1226-1231, 2013.

[54] D. S. Mangum, J. Downie, C. C. Mason et al., "VPREB1 deletions occur independent of lambda light chain rearrangement in childhood acute lymphoblastic leukemia," Leukemia, vol. 28, no. 1, pp. 216-220, 2014.

[55] A. K. Andersson, J. Ma, J. Wang et al., "The landscape of somatic mutations in infant $M L L$-rearranged acute lymphoblastic leukemias," Nature Genetics, vol. 47, no. 4, pp. 330-337, 2015.

[56] R. C. Harvey, C. G. Mullighan, X. Wang et al., "Identification of novel cluster groups in pediatric high-risk B-precursor acute lymphoblastic leukemia with gene expression profiling: correlation with genome-wide DNA copy number alterations, 
clinical characteristics, and outcome," Blood, vol. 116, no. 23, pp. 4874-4884, 2010.

[57] J. J. Yang, D. Bhojwani, W. Yang et al., "Genome-wide copy number profiling reveals molecular evolution from diagnosis to relapse in childhood acute lymphoblastic leukemia," Blood, vol. 112, no. 10, pp. 4178-4183, 2008.

[58] R. Y. L. Tsai and R. R. Reed, "Cloning and functional characterization of Roaz, a zinc finger protein that interacts with $\mathrm{O} / \mathrm{E}-1$ to regulate gene expression: implications for olfactory neuronal development," The Journal of Neuroscience, vol. 17, no. 11, pp. 4159-4169, 1997.

[59] L. E. Cheng, J. Zhang, and R. R. Reed, “The transcription factor Zfp423/OAZ is required for cerebellar development and CNS midline patterning," Developmental Biology, vol. 307, no. 1, pp. 43-52, 2007.

[60] R. Y. L. Tsai and R. R. Reed, "Identification of DNA recognition sequences and protein interaction domains of the multiple- $\mathrm{Zn}$ finger protein Roaz," Molecular and Cellular Biology, vol. 18, no. 11, pp. 6447-6456, 1998.

[61] A. Hata, J. Seoane, G. Lagna, E. Montalvo, A. HemmatiBrivanlou, and J. Massagué, "OAZ uses distinct DNA- and protein-binding zinc fingers in separate BMP-Smad and Olf signaling pathways," Cell, vol. 100, no. 2, pp. 229-240, 2000.

[62] M.-C. Ku, S. Stewart, and A. Hata, "Poly(ADP-ribose) polymerase 1 interacts with OAZ and regulates BMP-target genes," Biochemical and Biophysical Research Communications, vol. 311, no. 3, pp. 702-707, 2003.

[63] G. Masserdotti, A. Badaloni, Y. S. Green et al., "ZFP423 coordinates Notch and bone morphogenetic protein signaling, selectively up-regulating Hes5 gene expression," The Journal of Biological Chemistry, vol. 285, no. 40, pp. 30814-30824, 2010.

[64] S. Huang, J. Laoukili, M. T. Epping et al., "ZNF423 is critically required for retinoic acid-induced differentiation and is a marker of neuroblastoma outcome," Cancer Cell, vol. 15, no. 4, pp. 328-340, 2009.

[65] Y.-W. Cho, C.-J. Hong, A. Hou et al., "Zfp423 binds autoregulatory sites in p19 cell culture model," PLoS ONE, vol. 8, no. 6, Article ID e66514, 2013.

[66] R. K. Gupta, Z. Arany, P. Seale et al., "Transcriptional control of preadipocyte determination by Zfp423,” Nature, vol. 464, no. 7288, pp. 619-623, 2010.

[67] R. K. Gupta, R. J. Mepani, S. Kleiner et al., “Zfp423 expression identifies committed preadipocytes and localizes to adipose endothelial and perivascular cells," Cell Metabolism, vol. 15, no. 2, pp. 230-239, 2012.

[68] U. J. Yun, N. Song, D. K. Yang et al., "miR-195a inhibits adipocyte differentiation by targeting the preadipogenic determinator Zfp423," Journal of Cellular Biochemistry, vol. 116, no. 11, pp. 2589-2597, 2015.

[69] A. Hammarstedt, S. Hedjazifar, L. Jenndahl et al., "WISP2 regulates preadipocyte commitment and PPARgamma activation by BMP4," Proceedings of the National Academy of Sciences of the United States of America, vol. 110, no. 7, pp. 2563-2568, 2013.

[70] S. Kang, P. Akerblad, R. Kiviranta et al., "Regulation of early adipose commitment by Zfp521," PLoS Biology, vol. 10, no. 11, Article ID e1001433, 2012.

[71] S. Warming, P. Liu, T. Suzuki et al., "Evi3, a common retroviral integration site in murine B-cell lymphoma, encodes an EBFAZ-related Krüppel-like zinc finger protein," Blood, vol. 101, no. 5, pp. 1934-1940, 2003.
[72] W. A. Alcaraz, D. A. Gold, E. Raponi, P. M. Gent, D. Concepcion, and B. A. Hamilton, "Zfp423 controls proliferation and differentiation of neural precursors in cerebellar vermis formation," Proceedings of the National Academy of Sciences of the United States of America, vol. 103, no. 51, pp. 19424-19429, 2006.

[73] M. Chaki, R. Airik, A. K. Ghosh et al., "Exome capture reveals ZNF423 and CEP164 mutations, linking renal ciliopathies to DNA damage response signaling," Cell, vol. 150, no. 3, pp. 533548, 2012.

[74] J. N. Ingle, M. Liu, D. L. Wickerham et al., "Selective estrogen receptor modulators and pharmacogenomic variation in ZNF423 regulation of BRCA1 expression: individualized breast cancer prevention," Cancer Discovery, vol. 3, no. 7, pp. 812-825, 2013.

[75] H. M. Bond, M. Mesuraca, E. Carbone et al., "Early hematopoietic zinc finger protein (EHZF), the human homolog to mouse Evi3, is highly expressed in primitive human hematopoietic cells," Blood, vol. 103, no. 6, pp. 2062-2070, 2004.

[76] H. M. Bond, M. Mesuraca, N. Amodio et al., "Early hematopoietic zinc finger protein-zinc finger protein 521: a candidate regulator of diverse immature cells," International Journal of Biochemistry and Cell Biology, vol. 40, no. 5, pp. 848-854, 2008.

[77] F. Bernaudo, F. Monteleone, M. Mesuraca et al., "Validation of a novel shotgun proteomic workflow for the discovery of proteinprotein interactions: focus on ZNF521," Journal of Proteome Research, vol. 14, no. 4, pp. 1888-1899, 2015.

[78] L. Harder, G. Eschenburg, A. Zech et al., "Aberrant ZNF423 impedes B cell differentiation and is linked to adverse outcome of ETV6-RUNX1 negative B precursor acute lymphoblastic leukemia," Journal of Experimental Medicine, vol. 210, no. 11, pp. 2289-2304, 2013.

[79] S. M. Chambers, N. C. Boles, K.-Y. K. Lin et al., "Hematopoietic fingerprints: an expression database of stem cells and their progeny," Cell Stem Cell, vol. 1, no. 5, pp. 578-591, 2007.

[80] R. Gazit, B. S. Garrison, T. N. Rao et al., "Transcriptome analysis identifies regulators of hematopoietic stem and progenitor cells," Stem Cell Reports, vol. 1, no. 3, pp. 266-280, 2013.

[81] J. Riddell, R. Gazit, B. S. Garrison et al., "Reprogramming committed murine blood cells to induced hematopoietic stem cells with defined factors," Cell, vol. 157, no. 3, pp. 549-564, 2014.

[82] T. Mega, M. Lupia, N. Amodio et al., "Zinc finger protein 521 antagonizes early B-cell factor 1 and modulates the B-lymphoid differentiation of primary hematopoietic progenitors," Cell Cycle, vol. 10, no. 13, pp. 2129-2139, 2011.

[83] E. Matsubara, I. Sakai, J. Yamanouchi et al., "The role of zinc finger protein 521/early hematopoietic zinc finger protein in erythroid cell differentiation," The Journal of Biological Chemistry, vol. 284, no. 6, pp. 3480-3487, 2009.

[84] L. Salerno, C. Cosentino, G. Morrone, F. Amato, and B. D. MacArthur, "Computational modeling of a transcriptional switch underlying B-lymphocyte lineage commitment of hematopoietic multipotent cells," PLoS ONE, vol. 10, no. 7, Article ID e0132208, 2015.

[85] M. K. Lobo, C. Yeh, and X. W. Yang, "Pivotal role of early B-cell factor 1 in development of striatonigral medium spiny neurons in the matrix compartment," Journal of Neuroscience Research, vol. 86, no. 10, pp. 2134-2146, 2008.

[86] W. N. Addison, M. M. Fu, H. X. Yang et al., "Direct transcriptional repression of Zfp423 by Zfp521 mediates a bone morphogenic protein-dependent osteoblast versus adipocyte 
lineage commitment switch," Molecular and Cellular Biology, vol. 34, no. 16, pp. 3076-3085, 2014.

[87] M. Wu, E. Hesse, F. Morvan et al., “Zfp521 antagonizes Runx2, delays osteoblast differentiation in vitro, and promotes bone formation in vivo," Bone, vol. 44, no. 4, pp. 528-536, 2009.

[88] E. Hesse, H. Saito, R. Kiviranta et al., "Zfp521 controls bone mass by HDAC3-dependent attenuation of Runx2 activity," The Journal of Cell Biology, vol. 191, no. 7, pp. 1271-1283, 2010.

[89] R. Kiviranta, K. Yamana, H. Saito et al., "Coordinated transcriptional regulation of bone homeostasis by Ebf1 and Zfp521 in both mesenchymal and hematopoietic lineages," Journal of Experimental Medicine, vol. 210, no. 5, pp. 969-985, 2013.

[90] M. Mesuraca, O. Galasso, L. Guido et al., "Expression profiling and functional implications of a set of zinc finger proteins, ZNF423, ZNF470, ZNF521, and ZNF780B, in primary osteoarthritic articular chondrocytes," Mediators of Inflammation, vol. 2014, Article ID 318793, 11 pages, 2014.

[91] R. La Rocca, M. Fulciniti, T. Lakshmikanth et al., "Early hematopoietic zinc finger protein prevents tumor cell recognition by natural killer cells," The Journal of Immunology, vol. 182, no. 8, pp. 4529-4537, 2009.

[92] D. Kamiya, S. Banno, N. Sasai et al., "Intrinsic transition of embryonic stem-cell differentiation into neural progenitors," Nature, vol. 470, no. 7335, pp. 503-510, 2011.

[93] R. Spina, G. Filocamo, E. Iaccino et al., "Critical role of zinc finger protein 521 in the control of growth, clonogenicity and tumorigenic potential of medulloblastoma cells," Oncotarget, vol. 4, no. 8, pp. 1280-1292, 2013.

[94] N. Ohkubo, E. Matsubara, J. Yamanouchi et al., "Abnormal behaviors and developmental disorder of hippocampus in zinc finger protein 521 (ZFP521) mutant mice," PLoS ONE, vol. 9, no. 3, Article ID e92848, 2014.

[95] S. Lou, X. Pan, T. Huang et al., "Incoherent feed-forward regulatory loops control segregation of C-mechanoreceptors, nociceptors, and pruriceptors," Journal of Neuroscience, vol. 35, no. 13, pp. 5317-5329, 2015.

[96] M. J. Justice, H. C. Morse III, N. A. Jenkins, and N. G. Copeland, "Identification of Evi-3, a novel common site of retroviral integration in mouse AKXD B-cell lymphomas," Journal of Virology, vol. 68, no. 3, pp. 1293-1300, 1994.

[97] S. Warming, T. Suzuki, T. P. Yamaguchi, N. A. Jenkins, and N. G. Copeland, "Early B-cell factor-associated zinc-finger gene is a frequent target of retroviral integration in murine B-cell lymphomas," Oncogene, vol. 23, no. 15, pp. 2727-2731, 2004.

[98] T. Hiratsuka, Y. Takei, R. Ohmori et al., "ZFP521 contributes to pre-B-cell lymphomagenesis through modulation of the pre-Bcell receptor signaling pathway," Oncogene, 2015.

[99] K. E. Hentges, K. C. Weiser, T. Schountz, L. S. Woodward, H. C. Morse III, and M. J. Justice, "Evi3, a zinc-finger protein related to EBFAZ, regulates EBF activity in B-cell leukemia," Oncogene, vol. 24, no. 7, pp. 1220-1230, 2005.

[100] J. Hauer, A. Borkhardt, I. Sánchez-García, and C. Cobaleda, "Genetically engineered mouse models of human B-cell precursor leukemias," Cell Cycle, vol. 13, no. 18, pp. 2836-2846, 2014.

[101] K. Miyazaki, N. Yamasaki, H. Oda et al., "Enhanced expression of p210BCR/ABL and aberrant expression of Zfp423/ZNF423 induce blast crisis of chronic myelogenous leukemia," Blood, vol. 113, no. 19, pp. 4702-4710, 2009.

[102] L. van der Weyden, G. Giotopoulos, K. Wong et al., "Somatic drivers of B-ALL in a model of ETV6-RUNX1; Pax $5^{+/-}$ leukemia," BMC Cancer, vol. 15, article 585, 2015.
[103] N. Yamasaki, K. Miyazaki, A. Nagamachi et al., "Identification of Zfp521/ZNF521 as a cooperative gene for E2A-HLF to develop acute B-lineage leukemia," Oncogene, vol. 29, no. 13, pp. 1963-1975, 2010.

[104] T. Haferlach, A. Kohlmann, L. Wieczorek et al., "Clinical utility of microarray-based gene expression profiling in the diagnosis and subclassification of leukemia: report from the international microarray innovations in leukemia study group," Journal of Clinical Oncology, vol. 28, no. 15, pp. 2529-2537, 2010.

[105] S. Aibar, C. Fontanillo, C. Droste et al., "Analyse multiple disease subtypes and build associated gene networks using genomewide expression profiles," BMC Genomics, vol. 16, supplement 5, p. S3, 2015.

[106] Y. Aoki, T. Watanabe, Y. Saito et al., "Identification of CD $34^{+}$and CD34- Leukemia-initiating cells in MLL-rearranged human acute lymphoblastic Leukemia," Blood, vol. 125, no. 6, pp. 967980, 2015.

[107] V. P. Lavallée, I. Baccelli, J. Krosl et al., “The transcriptomic landscape and directed chemical interrogation of MLL-rearranged acute myeloid leukemias," Nature Genetics, vol. 47, no. 9, pp. 1030-1037, 2015.

[108] K. K. Fleischmann, P. Pagel, I. Schmid, and A. A. Roscher, "RNAi-mediated silencing of MLL-AF9 reveals leukemiaassociated downstream targets and processes," Molecular Cancer, vol. 13, no. 1, article 27, 2014.

[109] Y. Qiao, X. Wang, R. Wang et al., "AF9 promotes hESC neural differentiation through recruiting TET2 to neurodevelopmental gene loci for methylcytosine hydroxylation," Cell Discovery, vol. 1, article 15017, 2015.

[110] F. Barabé, J. A. Kennedy, K. J. Hope, and J. E. Dick, "Modeling the initiation and progression of human acute leukemia in mice," Science, vol. 316, no. 5824, pp. 600-604, 2007.

[111] J. Wei, M. Wunderlich, C. Fox et al., "Microenvironment determines lineage fate in a human model of MLL-AF9 leukemia," Cancer Cell, vol. 13, no. 6, pp. 483-495, 2008.

[112] S. J. Horton, J. Jaques, C. Woolthuis et al., "MLL-AF9-mediated immortalization of human hematopoietic cells along different lineages changes during ontogeny," Leukemia, vol. 27, no. 5, pp. 1116-1126, 2013.

[113] T. A. Schwickert, H. Tagoh, S. Gültekin et al., "Stage-specific control of early B cell development by the transcription factor Ikaros," Nature Immunology, vol. 15, no. 3, pp. 283-293, 2014.

[114] E. Bolton-Gillespie, M. Schemionek, H.-U. Klein et al., "Genomic instability may originate from imatinib-refractory chronic myeloid leukemia stem cells," Blood, vol. 121, no. 20, pp. 4175-4183, 2013. 


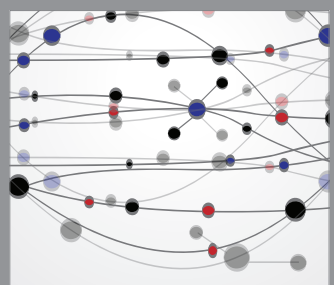

The Scientific World Journal
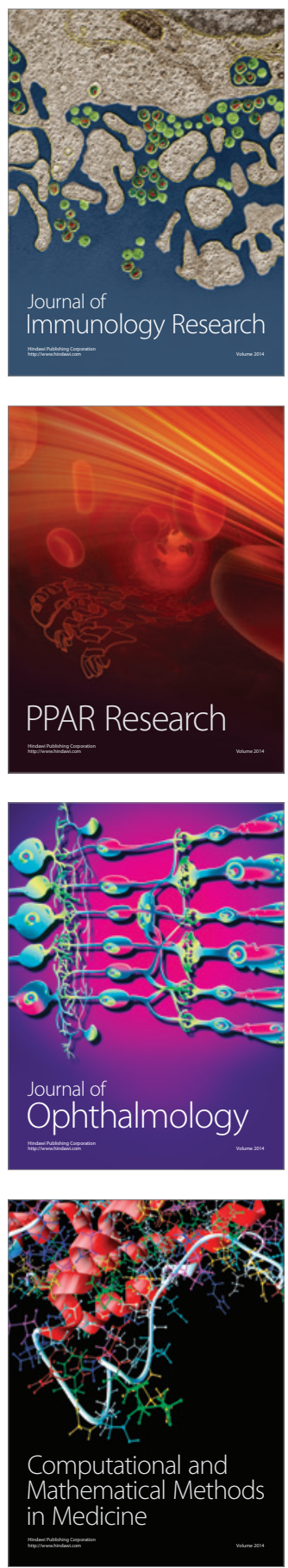

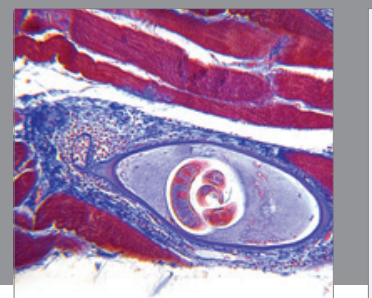

Gastroenterology

Research and Practice
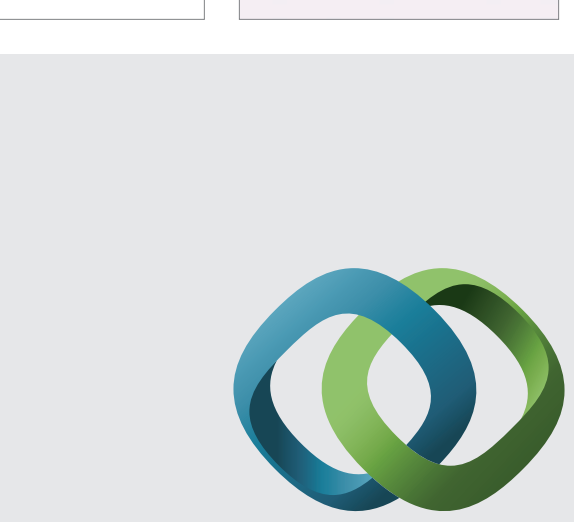

\section{Hindawi}

Submit your manuscripts at

http://www.hindawi.com
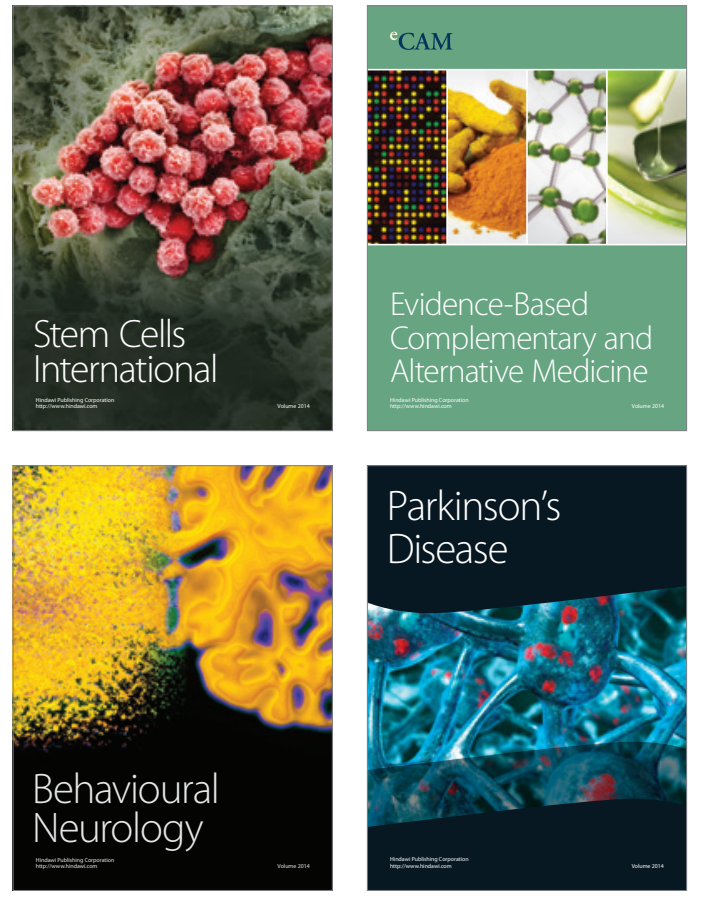
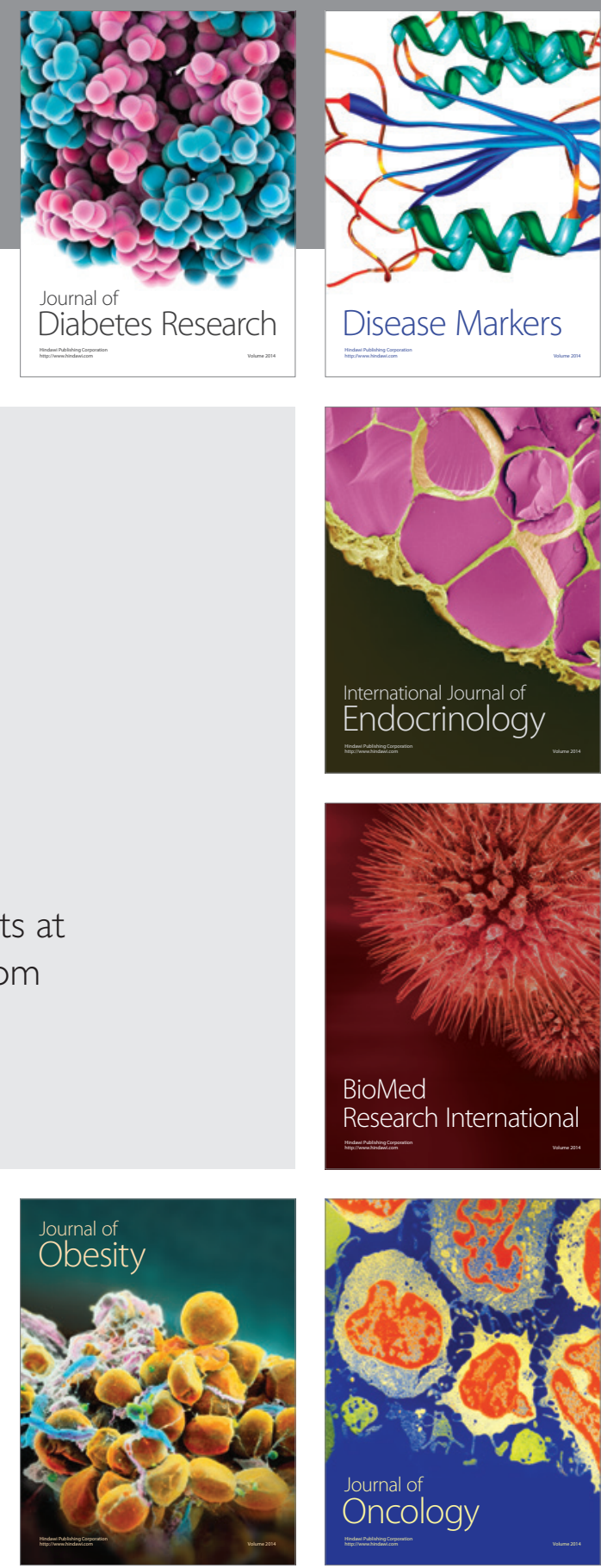

Disease Markers
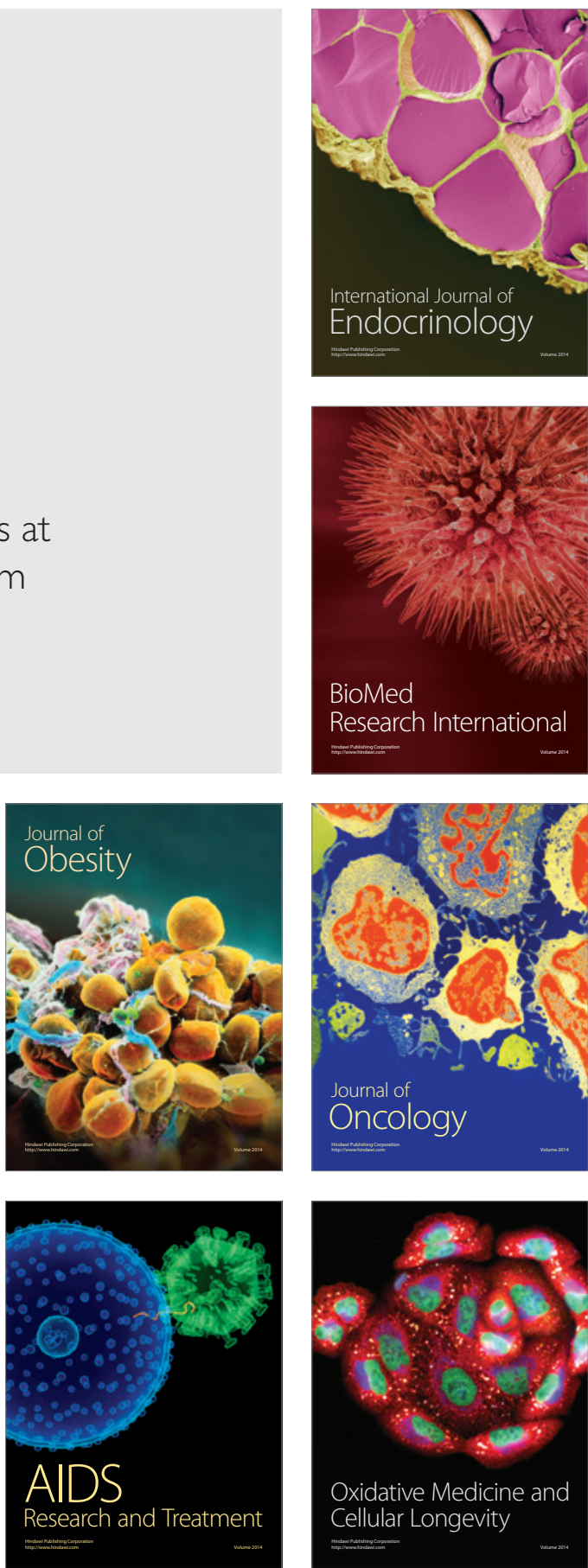